\title{
Quantitative characterization of microstructures formed during plastic deformation
}

\author{
A. Godfrey, ${ }^{*}$ X. Huang, ${ }^{* *}$ G. Winther, ${ }^{* *}$ Q. Liu*, and N. Hansen** \\ * Department of Materials Science and Engineering, Tsinghua University, Beijing 100084, China. \\ **Center for Fundamental Research: Metal Structures in Four Dimensions, Materials Research \\ Department, Risø National Laboratory, Roskilde DK 4000, Denmark.
}

During plastic deformation of a metal a fraction of the dislocations introduced remain stored in the microstructure. In many metals and alloys these dislocations collect into rotation boundaries, resulting in a process of microstructural subdivision during deformation. A quantitative understanding of the characteristics of deformation microstructures is important in order both to provide information about the physical mechanisms in operation during plastic deformation, and for use in the development of structure-property relationships. Some of the most important parameters of such microstructures include the dislocation boundary spacing, misorientation angle and axis, width, and inclination (boundary plane).

As the dislocation boundaries form a 3-dimensional microstructure, it is necessary to consider the limitations imposed by examination of sample sections where only 2-dimensional information is available. When this is done it is found that the distributions of boundary misorientation angles and boundary spacings exhibit a scaling behavior over a very wide strain range. The scaling analysis supports the idea that the observed dislocation boundaries can be divided into two classes [1]. Much useful information concerning the dislocation slip system activity can also be obtained from determination of the dislocation boundary plane, particularly for extended planar dislocation boundaries. Although images from thin-foils for examination in the transmission electron microscope (TEM) are a 2-dimensional projection of the thin-foil, the possibility of sample-tilting in the TEM allows the unique determination of the boundary plane from only a single sample. Extensive experimental investigations of TEM thin-foils show that for the planar boundaries, the boundary plane is more closely related to the crystallographic reference frame than to the macroscopic reference frame, and that for grains of certain orientations, the planar boundaries lie on, or near to a dislocation slip plane (Fig. 1) [2]. The choice of sample section is however important because in many cases two sets of planar boundaries co-exist, an optimal section exists where the traces of the each set of boundaries differ by a large angle, and where the boundaries are steeply inclined to the thin-foil. For rolling the optimal section is the plane containing the rolling direction and the normal direction.

Another area where it is particularly important to consider the full 3-dimensional characteristics of the microstructure is in determination of the stored energy of deformation. For many microstructures the stored energy can be estimated as the product of the boundary area per unit volume and the boundary energy per unit area, allowing measurement of the stored energy on a local microstructural length scale. An analysis shows that it is important to take into account the different geometric characteristics of the two classes of dislocation boundary, and to consider the distribution of boundary misorientation angles in making such calculations [3], particularly because the recrystallization behavior depends in a sensitive manner on the magnitude of the stored energy and on the heterogeneity of its distribution in the microstructure. If measurements are made using 
electron-back scatter pattern (EBSP) analysis, additional errors are introduced due the limited angular resolution of EBSP technique [4].

\section{References}

[1] A. Godfrey, D.A. Hughes, Scripta Materialia 51 (2004) 831.

[2] G. Winter et al., Acta Materialia 52 (2004) 4437.

[3] A. Godfrey et al., Proc. $25^{\text {th }}$ Risø International Symposium on Materials Science, Risø National Laboratory, Roskilde (2004) 317.

[4] This research was supported in part by the National Natural Science Foundation of China under contract no: 50371041. The Danish National Research Foundation is gratefully acknowledged for supporting the Center for Fundamental Research: Metal Structures in Four Dimensions.

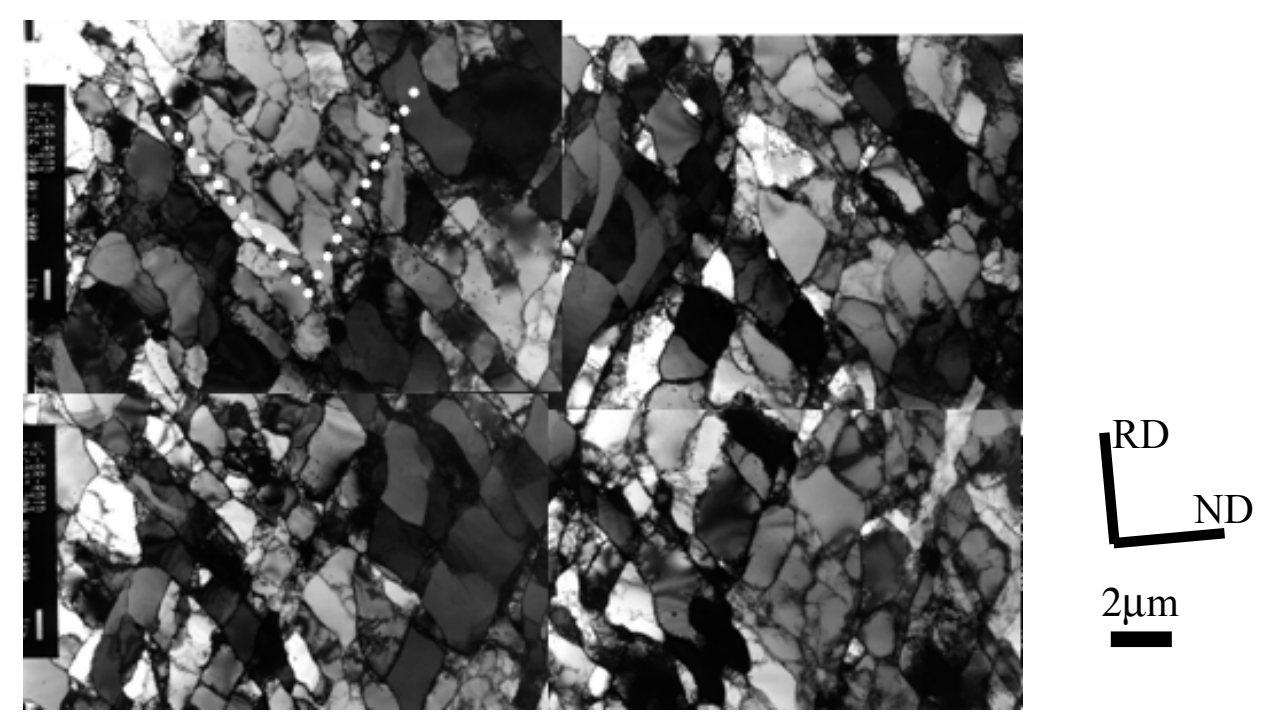

FIG. 1. TEM image of a $\{110\}<112>$ orientation Al single crystal deformed by channel die compression to a strain of $\varepsilon=0.5$. Dotted lines (in white) mark the traces of the active $\{111\}$ slip planes. Tilting of the sample such that the boundary traces remain sharp (edge-on) confirms that both sets of extended planar boundaries are parallel to the active $\{111\}$ slip planes.

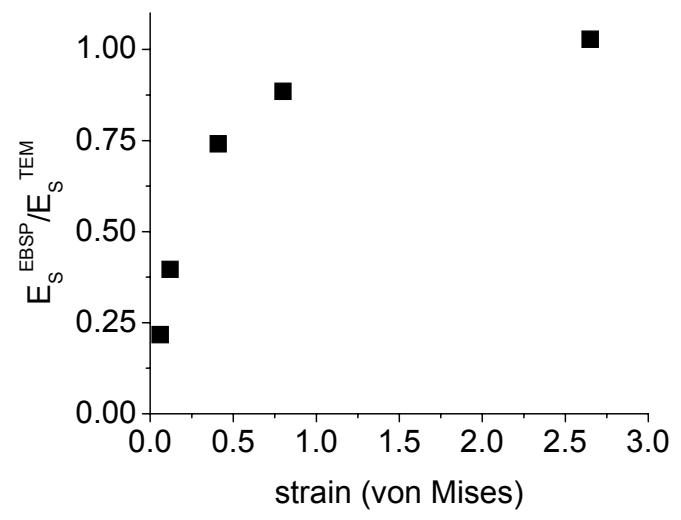

FIG. 2. Estimated error in calculated stored energy per unit volume due to EBSP limitations as a function of strain (data for rolled Al 99.99\%). $\mathrm{E}_{\mathrm{S}}{ }^{\mathrm{TEM}}$ = stored energy estimate from TEM data; $\mathrm{E}_{\mathrm{S}}{ }^{\mathrm{EBSP}}=$ stored energy based on simulated EBSP measurements with minimum resolution of $1.5^{\circ}$. 